Ann. Sci. For., 1986, 43 (2), 131-146

\title{
Comportement expérimental de semis de chêne pédonculé, chêne sessile et hêtre en présence d'une nappe d'eau dans le sol
}

\author{
G. LEVY, M. BECKER * et B. GARREAU ** \\ avec la collaboration technique de Y. LEFEVRE \\ INRA, Station de Recherches sur les Sols, la Microbiologie \\ et la Nutrition des Arbres Forestiers \\ * Laboratoire de Phytoécologie Forestière \\ Centre de Recherches forestieres de Nancy \\ Champenoux, F 54280 Seichamps \\ *:* Direction Départementale de l'Agriculture \\ Cité Administrative \\ Rue Jules-Ferry - B.P. 50-F 33090 Bordeaux Cedex
}

\section{Résumé}

Cette expérience en vases de végétation est destinée à comparer le comportement de semis de chêne pédonculé, chêne sessile et hêtre en présence de nappes situćes à différentes profondeurs dans le sol.

Lorsque la nappe est installée dès le semis, puis maintenue en place, on note les résultats suivants :

- une nappe très superficielle (à $-2 \mathrm{~cm}$ ) affecte plus la levée des chênes (environ 58 p. 100 de semis levés) que celle du hêtre $(74$ p. 100). Peu de semis meurent ensuite, et uniquement chez le hêtre;

- la croissance de $1^{\text {r. }}$ année, ainsi que la plupart des caractéristiques des plants mesurées en fin de seconde année, commencent à être affectées lorsque la nappe est située à environ $-5 \mathrm{~cm}$ pour le chêne pédonculé, $-8 \mathrm{~cm}$ pour les deux autres espèces :

- une fertilisation effectuée en début de $2^{\prime \prime}$ année n'a pas modifié sensiblement les seuils à partir desquels les caractéristiques des plants commencent à être affectées.

Lorsque la nappe est installée plus tardivement (alors que les semis présentent déjà un certain développement), tous les semis de hêtre meurent rapidenent en présence d'une nappe superficielle, alors que tous les semis de chêne résistent : le hêtre justific alors sa réputation d'espèce très sensible à l'engorgement.

Il est rappelé enfin que lc meilleur comportement des semis de chêne pédonculé par rapport à ceux de chêne sessile, mis en évidence ici, ne peut amener à choisir dans tous les cas le pédonculé pour la mise en valeur des sols à hydromorphie temporaire : dans certaines stations forestières (pseudogleys acides à texture grossière), il semble que la résistance à la sécheresse soit prépondérante par rapport à la résistance à l'engorgement dans le comportement global des arbres adultes.

Mots clés: Chéne péaonculé, chêne sessile, hêtre, engorgemunt, semis. 


\section{Introduction}

Le chêne pédonculé (Quercus robur L.) et le chêne sessile (Quercus petraea (Matt.) Liebl.) sont les essences forestières les plus répandues sur les sols à forte hydromorphie temporaire de la moitié Nord de la France; on peut donc penser qu'ils sont bien adaptés à un ennoyage périodique plus ou moins important de leur système racinaire. On constate cependant une forte diminution de production sur certains de ces sols, notamment les pélosol-pseudogleys, et surtout de fréquentes et très sérieuses difficultés pour la régénération naturelle des peuplements (levée et croissance initiale).

Des recherches effectuées en forêt ont permis de confirmer ce dernicr point : mauvaise germination et faible enracinement des semis de chêne après remontée des nappes [Pellecuer, 1976, en forêt de Morimond (52)]; effet propre positif de labaissement de la nappe par drainage sur la survie des semis de chêne sessile (INGRAIN, 1978, en forêt de Fontainebleau), et sur la survie et la vigucur des semis des deux espèces [BECKER \& LÉVY, 1983, en forêt de Mondon (54)].

Ces études ne permettent pas de comparer avec précision le comportement des dcux espèces. On pense souvent que le chêne pédonculé résisterait mieux que le sessile à la présence d'une nappe superficielle (JONES, 1959); il est cependant difficile de conclure avec certitude en raison des interactions qui peuvent intervenir dans le milicu naturel. Ainsi, les stations typiques du chêne pédonculé sont-elles situées dans des vallons où la nappe cst souvent proche de la surface : or, on remarque que ces sols bénéficient généralement d'une certaine fraîcheur estivale et d'une richesse minérale très satisfaisante.

En ce qui concerne ce dernier point, Pellecuer (1976) a constaté que la germination et la croissance initiale des semis des deux espèces apparaissaicnt d'autant plus importantes que le sol était plus riche et l'humus moins acide; cependant, le chêne sessile semblait plus affecté que le chêne pédonculé par la pauvreté chimique du sol, ce qui était d'ailleurs souvent en contradiction avec la répartition des semenciers.

Quant au hêtre, il semble bien qu’il résiste nettement moins que les chêncs à la présence d'une nappe temporaire dans le sol. Il est ainsi absent de la plupart des stations à hydromorphie superficielle et accentucee.

BELgRAND (1983) a étudié en conditions contrôlées l'influence de nappes situées à différentes profondeurs dans le sol, combinées ou non avec une fertilisation, sur le comportement de semis repiqués de chêne pédonculé, de chêne rouge (Quercus rubra L.) et de hêtre. Cet auteur a mis en évidence la très bonne résistance du chêne pédonculé, en particulier sur le plan de la survie mais aussi de la croissance dans la mesure où l'on avait préalablement effectué des apports d'azote; le hêtre s'est avéré par contre considérablement plus sensible à la présence d'une nappe.

Il n'existe cependant pas, à notre connaissance, d’étude expérimentale comparative de la résistance de jeunes plants de chêne pédonculé et de chêne sessile à la présence d'une nappe dans le sol. Une telle comparaison serait d'autant plus intéressante que ces deux espèces ont probablement une autécologic beaucoup moins semblable que ce qui était souvent admis jusquici : le récent dépérissement du chêne observé en France a en effet opéré une ségrégation tranchée entre le pédonculé (seul atteint) et le sessile (toujours indemne) (BECKER \& LÉVY, 1982). 
C'est pourquoi nous avons comparé en milieu contrôlé, depuis le semis jusqu'à la fin de la seconde année de végćtation, le comportement du chêne pédonculé, du chêne sessile et du hêtre (espèce de référence probablement très sensible) en présence d'une nappe située à différentes profondeurs dans le sol. L'influence éventuelle d'une

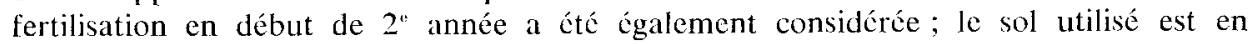
effet assez pauvre; c'est celui de la forêt de Mondon, où nous avons effectué l'étude sur la régénération des chênes citée précédemment (BECKER \& LF́vY, 1983).

\section{Matériel et méthodes}

\subsection{Mise en place}

L'expérience s'est déroulée en serre (sans éclairage ni chauffage d'appoint, sauf la mise hors gel), dans 90 vases de végétation cylindriques d'environ 201 , remplis de sol et munis d'un dispositif (décrit précédemment : LÉvY, 1981) permettant d'installer une nappe d'eau dans le sol et de la maintenir à la profondeur désirée. La terre a été prélevée en forêt de Mondon (54), dans l'horizon $A_{1}$ (mull acide) d'un sol brun lessivé à pseudogley.

Trente seaux sont consacrés à chaque essence. Vingt-deux semences ont été disposées dans chaque seau : glands de chêne pédonculé provenant de la forêt de Charmes (88), glands de chêne sessile de même provenance, faînes récoltées en forêt d'Eawy (76). Les glands ont été installés à la mi-février, les faînes début mars.

\subsection{Protocole de I"c année}

Il y a dix traitements. Pour les sept premiers, l'engorgement a cu lieu dès la misc en place des semences. Pour les trois derniers, lengorgement a été tardif et a débuté à la même date pour les trois espèces; celles-ci avaient alors atteint les stades de développement suivants : première pousse achevée pour les chênes; deux premières feuilles complètement développées pour le hêtre. Ces dix traitements sont les suivants :

- nappes non renouvelées à $-2,-6,-10,-18 \mathrm{~cm}$, soit 4 traitements, appelés $-2,-6,-10,-18$. Ce dernier $(-18)$ est considćré comme témoin bénéficiant de bonnes conditions hydriques;

- nappes à -2 et à $-6 \mathrm{~cm}$, avec renouvellement bi-hebdomadaire de l'eau, soit 2 traitements, appelés $-2 R$ et $-6 R$;

- nappe alternativement à -2 et à $-6 \mathrm{~cm}$ (variation hebdomadaire), soit 1 traitement, appelé $-2-6$;

- nappes à $-2,-6$ et $-10 \mathrm{~cm}$ (engorgement tardif), soit 3 traitements appelés $-2 T$, $-6 T$ et $-10 T$.

Il y a trois répétitions, soit 66 semences au total, par traitement.

Les nappes ont été supprimées le $01 / 07$ et les sols maintenus ensuite à la capacité au champ. 
Les relevés et mesures suivants ont été effectués : levée des semis, mortalité, observations sur la croissance des plants, hauteur au $01 / 07$, hauteur à la fin de la $1^{\text {re }}$ année.

\subsection{Protocole de $2^{*}$ année}

Nous nous sommes surtout intéressés au comportement de plants soumis au cours de deux années consécutives à des nappes précoces et non renouvelées, car il s'agit du cas le plus simple à interpréter. Les traitements $-2,-6,-10$ et -18 ont été ainsi appliqués aux mêmes vases de végétation qu'en première année, et sont désignés de la même façon.

Par ailleurs, et accessoirement, nous avons voulu voir siil y avait interaction entre engorgement et nutrition minérale, autrement dit si une fertilisation modifiait sensiblement les conclusions obtenues à partir des modalités précédentes. Les traitements de $1^{\mathrm{r}}$ année $-2-6,-2 R,-6 R$ et $-10 R$ ont ainsi subi en seconde année des nappes affleurant respectivement à $-2,-6,-10$ et $-18 \mathrm{~cm}$, après avoir été fertilisés ; ils seront appelés : $-2 \mathrm{~F},-6 \mathrm{~F},-10 \mathrm{~F}$ et $-18 \mathrm{~F}$.

Le tableau 1 donne la correspondance entre traitements de $1^{\text {r. }}$ et $2^{\text {" année. }}$

\section{TABLEAU 1}

Correspondance entre traitements de $1^{\text {re }}$ et $2^{r}$ année ( $R=$ renouvellement de l'eau, $T=$ nappe tardive, $F=$ fertilisation ).

Second year treatment for each first year treatment $(R=$ renewal of water. $T=$ water-table set up later. $F=$ fertilization).

\begin{tabular}{c|c|c|c|c|c|c|c|c|c|c}
\hline \hline $1^{\mathrm{r}}$ année & -2 & -6 & -10 & -18 & $-2 R$ & $-6 R$ & $-2-6$ & $-2 T$ & $-6 \mathrm{~T}$ & $-10 \mathrm{~T}$ \\
\hline $2^{\prime \prime}$ année. & -2 & -6 & -10 & -18 & $-6 \mathrm{~F}$ & $-10 \mathrm{~F}$ & $-2 \mathrm{~F}$ & & & $-18 \mathrm{~F}$ \\
\hline
\end{tabular}

Au début de la 2" année, nous n’avons conservé que 7 plants (représentatifs) dans chaque vase de végétation. Deux apports d'engrais ont été effectućs dans les vases concernés, le premier après gonflement du bourgeon terminal des plants (le 2 mars pour les hêtres, le 15 mars pour les chênes), le second une semaine plus tard ; chacun d'entre eux comprenait, par vase de végétation, $4 \mathrm{~g}$ d'ammonitrates (à $34 \mathrm{p}$. 100) et $50 \mathrm{ml}$ de solution de $\mathrm{PO}_{4} \mathrm{H}_{2} \mathrm{~K}$ (phosphate monopotassique) à $40 \mathrm{~g} / \mathrm{l}$. Nous avons installé les nappes la semaine suivante, après avoir lavé la terre pour éviter les excès de pression osmotique dus aux restes d'engrais.

Les vases ont été tous ressuyés le 17 juillet. L'expérience a pris fin courant septembre, et un certain nombre de variables ont été mesurées et exprimées en valeur moyenne par plant pour le vase de végétation considéré : hauteur totale, biomasse totale, poids du système racinaire, poids des racines fines ( $\leqslant 1 \mathrm{~mm}$ de diamètre), poids des feuilles de $2^{\circ}$ année, poids du système aérien, poids des tiges de 2 ans. 


\section{Résultats}

\subsection{Levée des semis}

\subsection{Au cours de la $I^{r r}$ année}

Elle est complète pour les traitements à engorgement tardif. Les résultats, pour les autres traitements, sont présentés dans le tableau 2. L'analyse de variance n'est significative que pour les deux espèces de chêne.

\section{Tableau 2}

Pourcentage de semis levés

[pour une même espèce, les traitements qui n'ont pas de lettre communte (a à $d$ ) sont statistiquement différents au seuil de $5 \mathrm{p}$. 100].

Percentage of seedlings sprung up [for a same species, treatments which have no letter in common (a to d) are statistically different].

\begin{tabular}{|c|c|c|c|c|c|c|c|}
\hline Traitements & -18 & -10 & $-6 R$ & -6 & $-2-6$ & $-2 R$ & -2 \\
\hline \multirow{2}{*}{ Chêne pédonculé } & 98,5 & 97,0 & 90,9 & 93,9 & 75,7 & 68,2 & 59,1 \\
\hline & a & a & a & a & b & $\mathrm{bc}$ & $\mathrm{c}$ \\
\hline \multirow{2}{*}{ Chêne sessile. } & 98,5 & 93,9 & 87,9 & 86,4 & 72.7 & 72,7 & 57,5 \\
\hline & a & $a b$ & $a b$ & $b c$ & $\mathrm{c}$ & c & d \\
\hline \multirow{2}{*}{ Hêtre } & 84,8 & 83,3 & 89,4 & 72,7 & 90,9 & 72,7 & 74,2 \\
\hline & a & a & a & a & $\mathbf{a}$ & a & a \\
\hline
\end{tabular}

On remarque en premier lieu que la levée n'est jamais inférieure à 55 p. 100 , même pour des nappes très superficielles $(-2 \mathrm{~cm})$.

La levée est pratiquement complète $(>90$ p. 100) pour le chêne pédonculé lorsque la nappe affleure à $-6 \mathrm{~cm}$ ou en dessous. Par contre, une nappe situće à $-2 \mathrm{~cm}$ (les glands sont alors pratiquement en contact avec l'eau) affecte significativement la levée. Les résultats sont comparables pour le chêne sessile, à la différence que la levée est déjà sensiblement affectée par une nappe à $-6 \mathrm{~cm}$.

L'effet d'un renouvellement de l'eau de la nappe n'est vraiment sensible que pour le niveau $-2 \mathrm{~cm}$ chez le chêne sessile.

Pour des nappes très superficielles $(-2 \mathrm{~cm})$, la levée est apparemment meilleure pour le hêtre que pour les chênes, ce qui peut paraître a priori surprenant. 
3.12. Survie des semis et phénomènes particuliers

\subsection{Les chênes}

Aucune mortalité n'intervient, quel que soit le traitement.

Certaines réactions à l'ennoyage apparaissent cependant, surtout pour les nappes les plus superficielles :

- la $1^{\text {re }}$ pousse d'un certain nombre de semis se dessc̀che; ce phénomène est plus fréquent chez le chêne sessile (tabl. 3). Rapidement cependant de nouvelles pousses se développent à partir de la base; elles atteignent toutes la même taille (pour un même plant);

- les feuilles sont plus petites, parfois nécrosées. La couleur des feuilles varie d'autre part en fonction du traitement : elles sont bien vertes pour - 18 et d'autant plus claires que la nappe est plus superficielle; cette différence de teinte s'accentue avec le temps.

\section{TABLEAU 3}

Dessèchement de la $1^{\text {re }}$ pousse des semis de chêne : pourcentage d'individus atteints.

Percentage of oaks seedlings affected by the first shoot drying.

\begin{tabular}{|c|c|c|c|c|c|c|c|}
\hline Traitements & -2 & $-2-6$ & -6 & -10 & -18 & $-2 R$ & $-6 R$ \\
\hline Chêne pédonculé & 15 & 12 & 11 & 2 & 0 & 14 & 9 \\
\hline Chêne sessile & 24 & 14 & 20 & 5 & 0 & 14 & 21 \\
\hline
\end{tabular}

\subsection{Le hêtre}

Presque tous les semis à engorgement tardif des traitements $-2 T$ et $-6 T$ sont morts. A l'installation de la nappe dans le traitement -27 , les feuilles se dessèchent brutalement, en l'espace d'une semaine, et les plants meurent. Le phénomène débute plus tardivement pour le traitement - 67', avec l'apparition de taches noires, qui s'étendent progressivement.

Pour les traitements à engorgement précoce, des nécroses peuvent apparaître sur le bord des feuilles 7 semaines après la germination (tabl. 4). Sur un petit nombre de plants, surtout pour le traitement -2 , le dessèchement peut gagner toute la feuille ; il arrive alors que le semis meure.

\subsection{Croissance des semis}

Des mesures de hauteur ont été effectuées au $1^{\text {tr }}$ juillet et à l'automne.

Les conclusions que l'on peut en tirer ćtant semblables, nous ne présenterons que les résultats de fin de saison. 


\section{TABleaU 4}

Nécrose des feuilles de hêtre : pourcentage d'individus atteints.

Percentage of beech seedlings affected by leaves drying.

\begin{tabular}{|c|c|c|c|c|c|c|c|}
\hline Traitements & -2 & $-2-6$ & -6 & -10 & -18 & $-2 R$ & $-6 R$ \\
\hline Semis atteints $(\%)$ & 44 & 38 & 20 & 3 & 0 & 32 & 17 \\
\hline
\end{tabular}

Les traitements $-2 T,-6 T$ et $-10 T$ n'ont pas modifié la hauteur finale des semis par rapport au témoin (-18) : presque tout l'allongement de l'année était en effet acquis au moment de l'engorgement.

Les hauteurs moyennes des semis, pour les autres traitements, sont présentées dans le tableau 5. L'analyse de variance indique des différences significatives pour les trois espèces.

\section{TABLEAU 5}

Hauteurs moyennes des semis (en $\mathrm{cm}$ ) en fin de $1^{\text {re }}$ année [analyse de variance : pour une même espèce, les traitements qui n'ont pas de lettre commune $\left(\begin{array}{lll}a & \text { à } & \text { d) }\end{array}\right.$ sont statistiquement différents au seuil de 5 p. 100].

Seedlings average height (in $\mathrm{cm}$ ) in the end of the first year [for a same species, treatments which have no letter in common (a to d) are statistically different].

\begin{tabular}{|c|c|c|c|c|c|c|c|}
\hline Traitements & -18 & -10 & $-6 R$ & -6 & $-2-6$ & $-2 R$ & -2 \\
\hline \multirow{2}{*}{ Chêne pédonculé } & 21,00 & 22,32 & 18,52 & 20,81 & 13,97 & 13,53 & 9,93 \\
\hline & a & $\mathrm{a}$ & a & $\mathbf{a}$ & $\mathrm{b}$ & $\mathrm{b}$ & $\mathrm{c}$ \\
\hline \multirow{2}{*}{ Chêne sessile } & 16,19 & 16,00 & 12,24 & 12,76 & 8,67 & 9,69 & 7,76 \\
\hline & $\mathrm{a}$ & a & $\mathrm{b}$ & b & $\mathrm{c}$ & c & c \\
\hline \multirow{2}{*}{ Hêtre } & 11,88 & 11,28 & 9,57 & 8,88 & 8,28 & 7,02 & 6,21 \\
\hline & a & a & $b$ & $b c$ & c & $d$ & d \\
\hline
\end{tabular}

Le renouvellement bi-hebdomadaire des nappes n'a dans l'ensemble pas eu d'effet très positif. Un renouvellement plus fréquent, mais lourd expérimentalement, aurait peut-être été plus efficace.

Pour le chêne pédonculé, on constate que le seuil à partir duquel la croissance a commencé à être affectée est compris entre les traitements -6 et $-2-6$, et doit 
donc être très proche de $-5 \mathrm{~cm}$. Quant au chêne sessile et au hêtre, leur croissance a déjà été affectée par le traitement -6 mais non par le traitement -10 ; pour ces deux espèces, le seuil correspond donc à une nappe plus profonde que dans le cas du chêne pédonculé, probablement voisine de $-8 \mathrm{~cm}$.

C'est donc indéniablement le chêne pédonculé qui se comporte le mieux vis-à-vis des nappes assez superficielles pour le critère «croissance de $1^{\text {re }}$ année ». Les deux autres espèces ont un comportement assez comparable; le hêtre semble cependant présenter un léger avantage : si le rapport entre la croissance du témoin $(-18)$ et celle du traitement - 6 est sensiblement le même $(1,27$ pour le chêne sessile et 1,34 pour le hêtre), il n'en est pas de même si l'on considère $-2-6$ au lieu de -6 : 1,87 et 1,43 respectivement.

La nappe la plus superficielle $(-2 \mathrm{~cm})$ affecte d'une manière peu différente la croissance des trois espèces : le rapport entre la croissance du témoin et celle du traitement -2 est, respectivement pour les chênes pédonculé et sessile et pour le hêtre, de 2,11, 2,09 et 1,91.

\subsection{Résultats en fin de seconde amnée}

\subsection{Traitements non fertilisés}

La figure 1 représente la variation relative de chacune des variables mesurées en fonction de la profondeur de la nappe. Ne sont pas représentées :

- la longueur de la pousse terminale de $2^{*}$ année, car les interprétations statistiques fournissent les mêmes résultats que pour la hauteur totale;

- trois des variables pour le hêtre, car les valeurs obtenues sont très faibles et peu fiables.

Les moyennes entre deux niveaux (successifs ou non) pour une même espèce ont été comparées par le test $t$ appliqué à des séries appariées.

On constate qu'en règle générale, les résultats obtenus en fin de $2^{\circ}$ année sont en accord avec ceux obtenus pour la hauteur en fin de $1^{\text {re }}$ année : pour le chêne pédonculé, seule une nappe plus superficielle que $-6 \mathrm{~cm}$ affecte significativement les variables, alors que pour les deux autres espèces, le traitement - 6 fournit déjà des résultats inférieurs au traitement -10 .

Il est difficile de déterminer la profondeur exacte à partir de laquelle une nappe devient néfaste ; seules quelques profondeurs ont en effet été prises en compte dans l'expérimentation; de plus, ce n'est pas parce qu'une différence entre les traitements - 10 et -6 , par exemple, n'est pas significative, que ce seuil ne peut pas être situé un peu au-delà de $-6 \mathrm{~cm}$ : l'aspect de la droite entre -10 et $-6 \mathrm{~cm}$ (selon qu'elle se rapproche de l'horizontale ou qu'elle présente une certaine pente) pourra donner des indications, mais sous toutes réserves. C'est ainsi qu'ici on peut supposer que les seuils à partir desquels ces variables commencent à être affectées par la nappe sont très voisins de $-5 \mathrm{~cm}$ pour le chêne pédonculé et de $-8 \mathrm{~cm}$ pour les deux autres espèces, comme dans le cas de la pousse de $1^{\text {re }}$ année.

Certaines variables font exception; pour elles, une différence significative entre les traitements -18 et -6 semble montrer que des nappes plus profondes pourraient commencer à être néfastes. Il s'agit en particulier : 
- pour les chênes, du poids des racines : celles-ci semblent plus sensibles à la nappe que les parties aériennes, au moins au cours des premières années;

- pour le hêtre, de la hauteur en fin de $2^{\circ}$ année : le hêtre serait alors plus sensible à la nappe que le chêne sessile pour la croissance aérienne, ce qui n'était pas le cas un an avant.

Les tests statistiques effectués pour comparer les espèces entre elles (comparaison des rapports entre les valeurs à $-18 \mathrm{~cm}$ et celles à $-6 \mathrm{~cm}$ ou $-2 \mathrm{~cm}$ ) confirment ce qui précède : le chêne sessile et le hêtre sont plus affectés que le chêne pédonculé par une nappe à $-6 \mathrm{~cm}$, et ceci pour l'ensemble des variables mesurées (sauf pour le poids du système racinaire du chêne sessile). Ils montrent par ailleurs

A
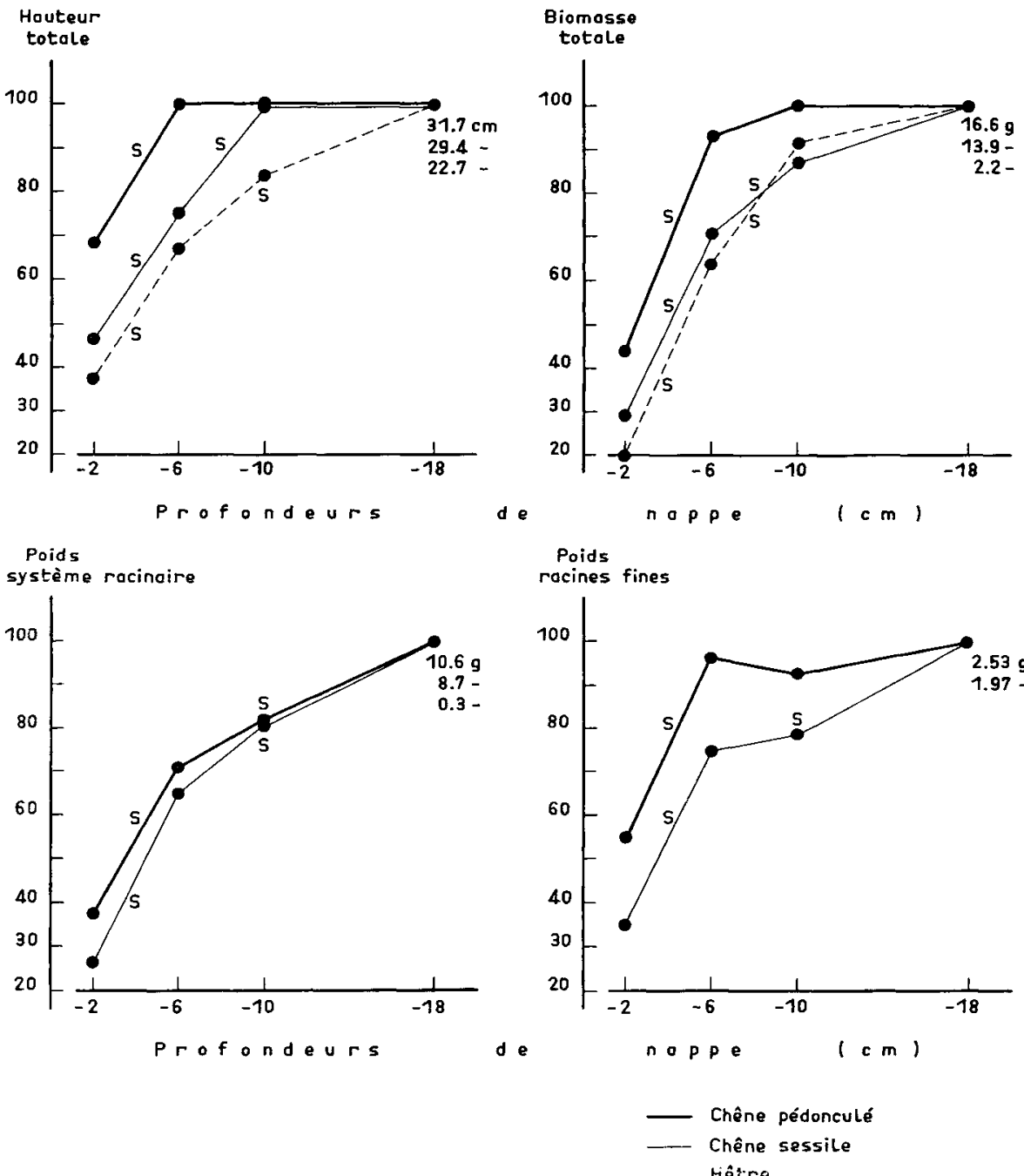
B
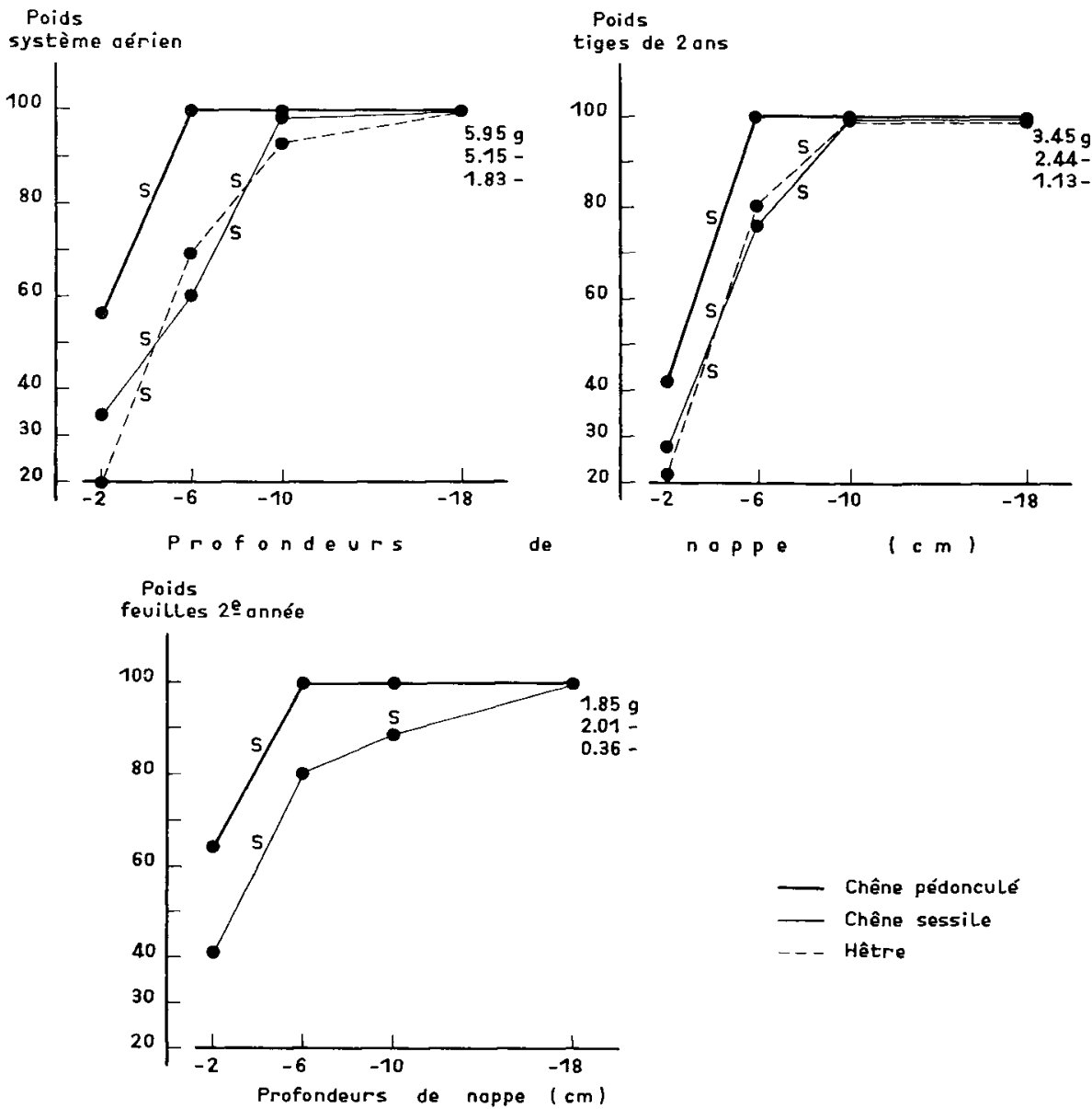

FIG. 1

Variation relative de différentes caractéristiques des plants en fonction de la profondeur de la nappe, pour la série non fertilisée, à la fin de l'expérience (moyennes par plants).

Relative variation of the plants various characteristics related to the water-table depth, for the non-fertilized series, in the end of the experiment (averages per plant).

- La valeur prise pour une nappe à $-18 \mathrm{~cm}$ est ramenée à 100 . La valeur réelle de la variable à ce niveau est indiquée en haut et à droite de chaque graphique (les 3 chiffres correspondent respectivement aux chênes pédonculé et sessile et au hêtre).

- La lettre $《 S 》$ correspondant à une nappe à $-4,-8$ ou $-10 \mathrm{~cm}$ indique une différence significative (at 5 p. 100) entre les valeurs que prend la variable pour des nappes respectivement a -2 et $-6 \mathrm{~cm},-6$ et $-10 \mathrm{~cm}$, ou -6 et $-18 \mathrm{~cm}$.

- The value of each characteristic for a water-table at - $18 \mathrm{~cm}$ is brought to 100 ; the real value for this depth is written in the upper right corner of each graph (the 3 numbers respectively correspond to the pedunculate oak, the sessile oak and the beech).

- The letter $" S$ » corresponding to a water-table at $-4,-8$ or $-10 \mathrm{~cm}$ means a significant difference (at $5 \mathrm{p} .100)$ between the values of the characteristic for watertables respectively at -2 and $-6 \mathrm{~cm},-6$ and $-10 \mathrm{~cm}$, or -6 and $-18 \mathrm{~cm}$. 
qu'il en est sensiblement de même pour une nappe à $-2 \mathrm{~cm}$. D'autre part, le hêtre semble relativement plus affecté que le chêne sessile par le traitement -2 , mais les différences ne sont généralement pas significatives.

Pour la plupart des variables mesurées, on ne connaît pas la part respective de chacune des deux années dans ces résultats finaux. L'examen de l'évolution de la croissance en hauteur semble cependant montrer que les mêmes tendances générales se sont maintenues durant toute l'expérience.

\subsection{Traitements fertilisés}

Rappelons qu'il ne s'agit que de traitements accessoires et dont les résultats doivent être examinés prudemment car les niveaux de nappe ont été modifiés par rapport à la $1^{\text {re }}$ année de l'expérience. Aussi nous contenterons-nous d'indiquer brièvement les résultats globaux.

Il apparaît en premier lieu que si, dans l'ensemble, les deux espèces de chênes ont fortement bénéficié de la fertilisation, celle-ci a par contre eu un effet généralement dépressif sur le hêtre, qui s'est ainsi peu développé en seconde année ; il apparaît donc impossible dans ces conditions d'interpréter les résultats relatifs à cette espèce.

Chez les deux espèces de chênes, l'examen des rapports entre chaque traitement fertilisé et le traitement correspondant non fertilisé paraît indiquer (compte tenu des traitements hydriques respectifs de 1 " année) que la fertilisation n'est pas plus efficace lorsque la nappe est superficielle que lorsqu'elle est plus profonde ; l'influence néfaste de l'engorgement ne semble aucunement due à un abaissement consécutif du niveau de nutrition minérale, du moins sur le sol pauvre utilisé et pour les profondeurs de nappe testées.

La conclusion globale essentielle qui se dégage des mesures de fin de seconde année est très comparable à celle émise dans le cas des traitements non fertilisés : le chêne pédonculé réagit mieux à la présence de nappes superficielles que le sessile.

Pour l'ensemble des caractéristiques étudiées sur les plants, il apparaît une différence significative entre 2 niveaux de nappe successifs :

- entre les traitements - 10 et -6 pour le sessile ;

- entre les traitements -6 et -2 pour le pédonculé (ainsi qu'entre -10 et - 6 pour le poids moyen du système racinaire, comme dans le cas des traitements non fertilisés).

Pour pratiquement toutes les variables, une nappe à $-6 \mathrm{~cm}$ affecte plus le sessile que le pédonculé (par rapport à la valeur correspondante du traitement - 18) (tabl. 6). Il en est de même pour une nappe à $-2 \mathrm{~cm}$, mais la différence entre les deux essences est plus faible qu’à $-6 \mathrm{~cm}$.

L'ensemble de ces résultats correspond-il cependant à des différences qui se sont développées au cours de la seconde année de l'expérience, après fertilisation (et non uniquement à des différences qui existaient déjà un an avant) ? La comparaison des hauteurs totales finales et des hauteurs correspondantes en fin de $1^{\text {ro }}$ année permet de répondre par l'affirmative pour cette variable; on peut penser au vu de l'ensemble des résultats de l'expérience, et en raison notamment de la forte influence positive générale de la fertilisation, qu'il en est de même pour les autres variables. 


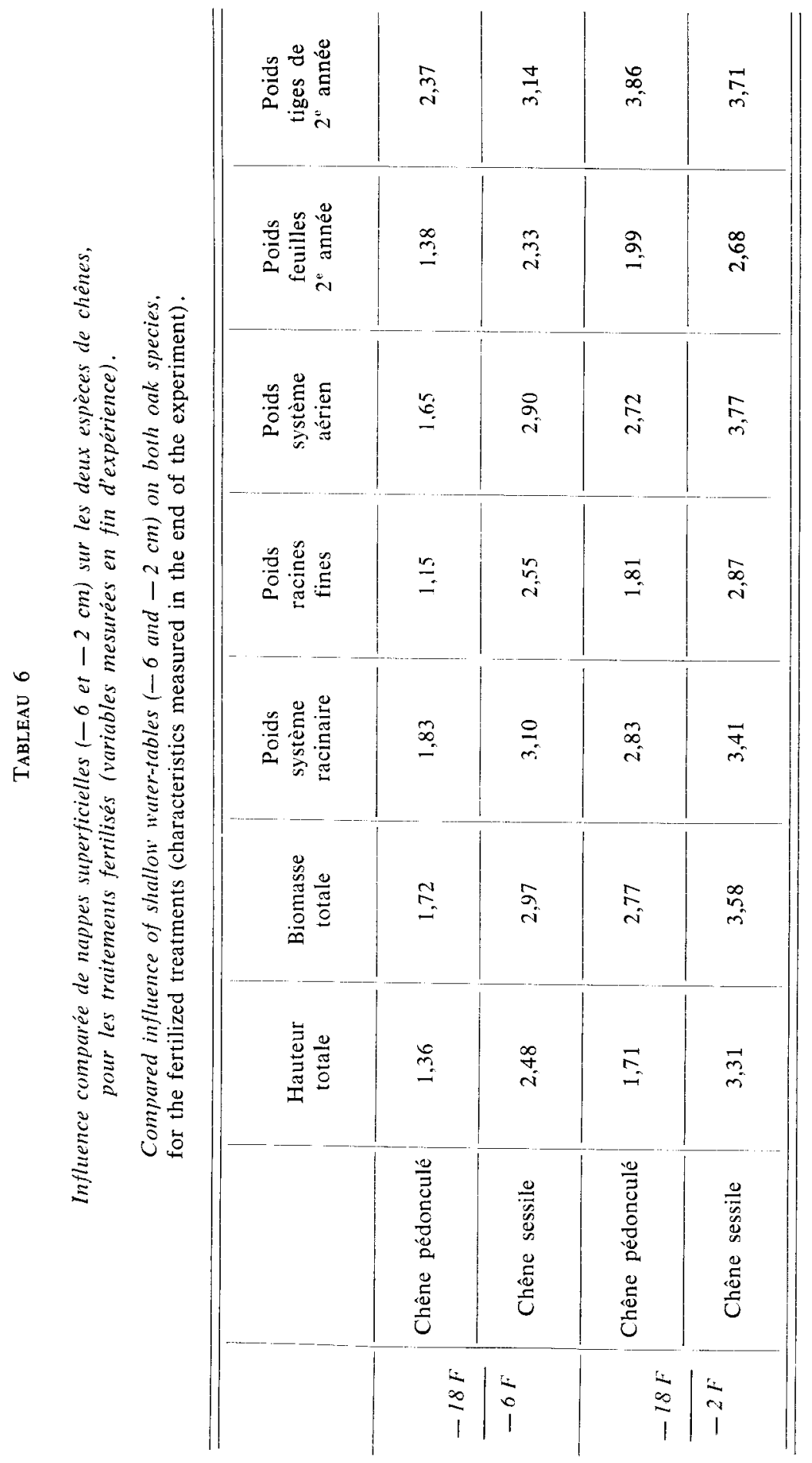




\section{Discussion, conclusion}

Bien que les résultats ne soient évidemment pas totalement transposables sans vérification préalable à des conditions autres que celles dans lesquelles s'est déroulée l'expérience (par exemple, les caractéristiques du sol), les points suivants peuvent être dégagés.

Dans la comparaison du comportement du hêtre avec celui des chênes, il importe de bien distinguer nappe précoce (mise en place dès le semis) et nappe tardive. Dans le premier cas, le hêtre n'apparaît pas significativement plus sensible à l'engorgement que le chêne sessile, tant pour sa croissance de première année que pour les caractéristiques étudiées en fin de seconde année. Si l'on constate des mortalités parmi les semis de hêtre, alors que tous les semis de chêne survivent, ce phénomène est relativement limité ; de plus, la germination du hêtre est moins affectée par la présence d'une nappe très superficielle que celle des chênes.

Par contre, dans le cas où la nappe n'est installée près de la surface que tardivement, alors que les semis présentent déjà un certain développement, la réputation du hêtre de forte sensibilité à l'engorgement est confirmée : il ne subsiste aucun plant de cette espèce pour les traitements $-2 T$ (le phénomène se produit alors en quelques jours) et $-6 T$, alors qu'aucune mortalité n'affecte les semis de chêne dans ces conditions. Le hêtre ne résiste done pas à l'apparition soudaine d'une nappe superficielle, alors que la présence permanente d'une nappe depuis le semis permet une adaptation progressive.

Ces résultats peuvent expliquer, au moins partiellement, l'absence du hêtre de la plupart des stations forestières à forte hydromorphie. La fluctuation des nappes varie en effet en liaison directe avec les conditions climatiques de l'année; un engorgement superficiel, subsistant quelques jours, peut fort bien apparaître tardivement certaines années. De plus le hêtre, débourrant généralement plus précocément que le chêne, est d'autant plus exposé à un ennoyage de son système racinaire en période de feuillaison.

Par ailleurs, le comportement du chêne pédonculé est meilleur que celui du sessile. C'est ainsi que pour presque toutes les variables mesurées, le pédonculé est insensible à une nappe à $-6 \mathrm{~cm}$, au contraire du sessile. Pour des nappes encore plus superficielles $(-2 \mathrm{~cm})$, le pédonculé est également relativement moins atteint. Seul le poids du système racinaire en fin de $2^{\prime \prime}$ année fait exception à ces résultats : il commence déjà à être affecté pour des nappes plus profondes ; de plus, la différence entre les deux espèces de chêne n'apparaît plus significative pour cette variable.

Une fertilisation préalable améliore très nettement l'ensemble des caractères mesurés sur les plants des deux espèces de chêne. Le comportement relatif de ces espèces vis-à-vis d'une nappe superficielle demeure cependant le même qu'en l'absence de fertilisation : les résultats concernant la sensibilité à l'hydromorphie semblent donc transposables à des sols plus riches que celui utilisé dans cette expérience. II apparaît également qu'il n'y a pas d'interaction, dans les conditions de l'expérience, entre la nappe et la nutrition minérale : l'action néfaste de la nappe ne se manifeste pas, ne serait-ce qu'en partie semble-t-il, par l'intermédiaire d'une modification de la nutrition minérale des plants. 
Ces résultats ne concordent pas entièrement avec ceux de Belgrand (1983). Pour cet auteur, une nappe à $-6 \mathrm{~cm}$ provoquait déjà une diminution du développement du chêne pédonculé, notamment de sa croissance en hauteur ; en fait, certaines conditions plus défavorables que dans notre expérience peuvent expliquer cette différence : la texture du sol était plus fine (une même nappe était donc plus défavorable, du fait d'un potentiel d'oxydo-réduction plus bas), et il s'agissait de plants repiqués et non de semis (ce qui entraînait l'ennoyage d'une partie du système racinaire préexistant).

Les résultats de l'expérience rapportée ici ne peuvent bien sûr servir de critère unique pour la mise en valeur forestière des sols hydromorphes. En effet, les arbres peuvent être soumis en forêt à des conditions hydriques au niveau du sol très variables au cours de l'année.

C'est ainsi que dans certaines conditions, le chêne sessile adulte présente un comportement nettement meilleur que le pédonculé sur des sols à hydromorphie très prononcée. C'est le cas en particulier des pseudogleys acides développés sur matériaux à texture assez grossière, donc à capacité de rétention en eau limitée ; ce comportement plus favorable du sessile se manifeste au niveau de la croissance, comme en Forêt de Mondon (Becker \& Lévy, 1986), ou même de la survie : ce fut le cas lors du dépérissement observé en forêt de Tronçais à la suite de la sécheresse de 1976, dépérissement dont seul le pédonculé a été victime (BECKER \& LÉVY, 1982). On peut penser que dans de telles conditions, laspect « résistance à la sécheresse » s'avère prépondérant par rapport à l'aspect « résistance à l'engorgement ». Par ailleurs, le fait qu'il s'agisse là d'arbres adultes, et non de jeunes semis comme dans notre expérience en serre, a peut-être aussi son importance (développement différent du système racinaire en profondeur et/ou modification du comportement écophysiologique avec l'âge?).

Reçu en mars 1985.

Accepté en juillet 1985.

\begin{abstract}
Summary
Compared behaviour of pedunculate oak, sessile oak and beech seedlings in the presence of a water-table in the soil : experimental study
\end{abstract}

The three species behaviour in the presence of water-table set at various depths in the soil, is compared during their first two years of life.

When the water-table is set up at the same time as the seeding, then maintained, the main results are :

- the oaks germination is more damaged (about 58 p. 100 of the seeds spring up) than that of the beech $(74$ p. 100) by a shallow water table $(-2 \mathrm{~cm})$ (table 2$)$. Few seedlings die afterwards, and only beech ones;

- the first year height growth, as well as most plants characteristics measured in the end of the second year (total height, total biomass, aerial system weight, second year leaves weight, 2-year-old stems weight) begin to be affected for a water table set at a depth of about $-5 \mathrm{~cm}$ in the case of the pedunculate oak, $-8 \mathrm{~cm}$ in the case of the other two species (table 5 and figure 1). The oaks root system however begin to be affected by deeper water-table:

-- a fertilization carried out at the beginning of the second ycar is very beneficial to both oak species wherever the water-table was set, but doesn't significantly alter the thresholds from where the plants characteristics begin to be affected. 
When the water-table is set up later (when the seedlings already show some development), all beech seedlings die in a few days when the water table is shallow, whereas all oaks seedlings survive : the beech reputation of being very sensitive to waterlogging is checked in that case.

However, the better behaviour of the pedunculate oak young plants, compared to that of the sessile oak shown in that experiment cannot lead to favour in all cases the pedunculate oak instead of the sessile oak on soils with temporary water-table : in some forest sites (acid pseudogleys with coarse texture), it seems that drought resistance is more important than waterlogging resistance for the growth and survival of adult trees.

Key words : Pedunculate oak, sessile oak, beech, waterlogging, seedlings.

\section{Références bibliographiques}

Becker M., Lévy G., 1982. Le dépérissement du chêne en forêt de Tronçais. Les causes écologiques. Ann. Sci. For., 39 (4), 439-444.

Becker M., Lévy G., 1983. Installation ế dynamique d'une population de semis de chêne en milieu hydromorphe sous l'influence de divers facteurs (lumière, régime hydrique, compétition herbacée). Acta Oecologica, Oecol. Plant., 4 (18), 3, 299-317.

Becker M., Lévy G., 1986. Croissance radiale comparée de chênes adultes (Quercus robur L. et Quercus petraea (Matt.) Liebl.) sur sol hydromorphe acide. Effet du drainage. Acta Oecologica, Oecol. Plant., 7 (21), 2, 123-143.

Belgrand M., 1983. Comportement de jeunes plants feuillus sur substrat ennoyé. Adaptations racinaires. Application à la mise en valeur forestière des pseudogleys. Thèse de Docteur-Ingénieur, I.N.A. Paris-Grignon, 188 p.

INGRAIN P.R., 1978. Germination, croissance et survie des semis de chône sessile en forêt de Fontainebleat. Symposium «Feuillus précieux», I.U.F.R.O., INRA Nancy-Champenoux, $100-110$.

Jones E.W., 1959. Biological flora of the British Isles : Quercus L. J. Ecol., 47, 169-222.

LÉvy G., 1981. Comportement de jeunes plants d'épicéa commun en sol à engorgement temporaire de surface : influence de divers facteurs du milieu. Ann. Sci. For.. 38 (1). 3-30.

Pellecuer B., 1976. L'écologie des chênes en forêt de Morimond et ses applications sylvicoles. Thèse de Docteur-Ingénieur, Université de Nancy I, 173 p. 